\title{
Energy Filtered Nano-diffraction Application in Growth Orientation Analysis of Magnetic Thin Films
}

\author{
Lifan Chen and Haifeng Wang
}

Materials Characterization, Western Digital, 44100 Osgood Road, Fremont, CA 94539

The new generation of perpendicular recording used for recording density of $100 \mathrm{~Gb} / \mathrm{in}^{2}$ and beyond has the bit size is on the order of a hundred nanometers. Shrinking bit size requires the media to have high coercivity, large anisotropy and small grain sizes (10 $\mathrm{nm}$ or smaller). Therefore, characterizing grain orientation and correlating to growth mechanism for engineering optimization with statistical significance becomes increasingly challenging.

Microstructural characterization of CoPtCr films deposited by DC magnetron sputtering has shown that the microstructure of CoPtCr thin film is composed of amorphous matrix and crystallites with grain size of about $7 \mathrm{~nm}$, as shown in Figure 1. As different sputtering processes have different dynamics for the formation of the nanostructure, it is very important to identify the structure and growth orientation of the crystallites. In this paper we describe a detailed investigation of the growth structure of the crystallites in CoPtCr films by semi-automated nano-diffraction. Such knowledge of the crystal structure will provide essential information for engineering and fabrication of multilayer materials and understanding film magnetic properties $[1,2,3]$.

Tecnai TF20 STEM/TEM (FEI Company) equipped with a Gatan Image Filter (GIF) system was used for this study in which both TEM and STEM are performed at $200 \mathrm{kV}$. In order to acquire a series of semi-automated energy filtered nano-diffraction patterns, a STEM microprobe needs to be controlled synchronously with a GIF CCD camera for pattern recording.

The growth orientation dataset of the $\mathrm{CoPtCr}$ film stack obtained by the semi-automated energy filtered nano-diffraction technique is shown as a series of diffraction patterns from each individual layer of the film stack in Figure 2. A typical CoPtCr film stack is composed of amorphous substrate/soft underlayer/magnetic layer/surface capping layer. In this study, we mainly focus on the magnetic layer, i.e., NiW/Ru/CoPtCr. Results show that the seed layer $\mathrm{NiW}$ (f.c.c.) is strongly $<111>$ textured. The $\mathrm{Ru}$ (h.c.p.) layer and $\mathrm{CoPtCr}$ (h.c.p.) magnetic layer present an epitaxial growth with $<001>$ growth texture. This implies that $\mathrm{NiW}_{\text {(f.c.c) }}, \mathrm{Ru}_{\text {(h.c.p.), }}$ and $\mathrm{CoPtCr}_{\text {(h.c.p.) }}$ layers share a common orientation in which the close-packed plans are parallel: $(111)_{\mathrm{NiW}} / /(002)_{\mathrm{Ru}} / /(002)_{\mathrm{CoPtCr}}$. This configuration enables the surface energy between the phases to be at the lowest, therefore thermodynamically favored.

\section{References}

[1] J.C.H. Spence and J.M. Zuo, Electron Microdiffraction, New York: Plenum Press, 2009.

[2] J.M. Cowley, Ed. Electron Diffraction Techniques, Vol. 1. New York: Oxford University Press, 2003.

[3] C. Dwyer and A.I. Kirkland, Applied Physics Letters, 90 (2007) 151104. 


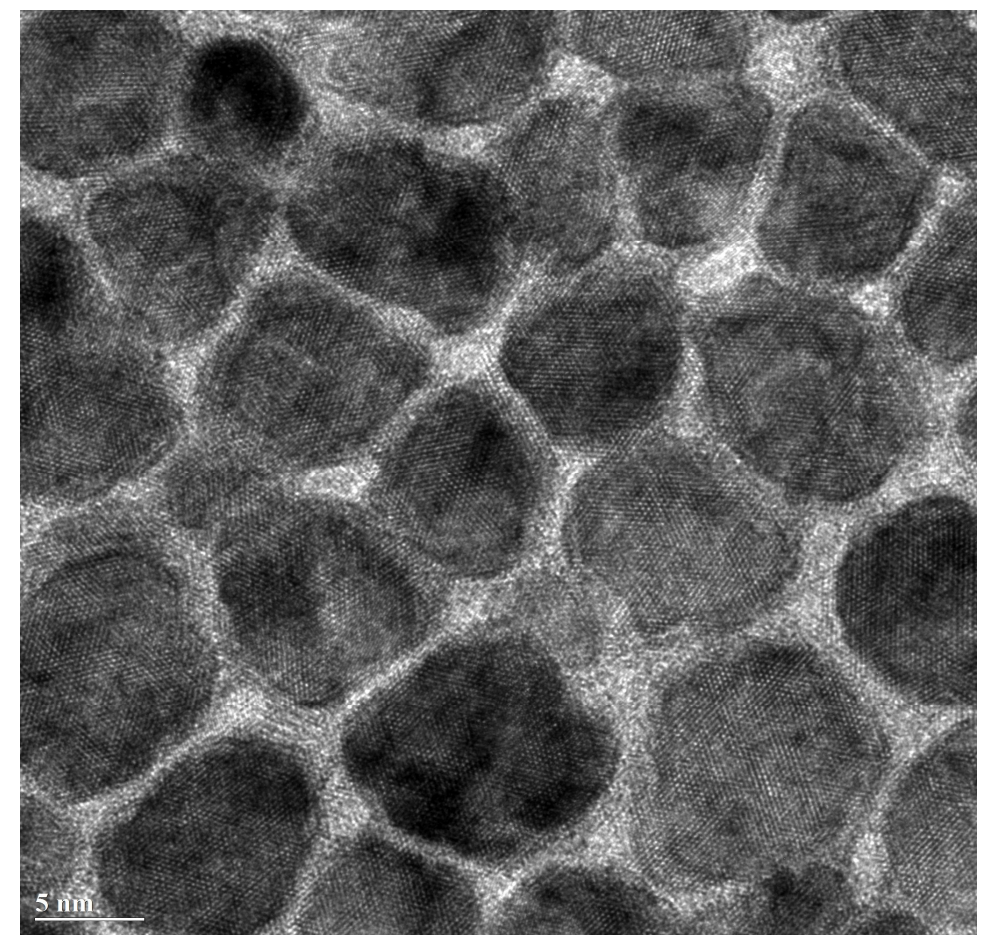

FIG. 1. Plan-view HRTEM micrograph showing the crystallites in the CoPtCr film.
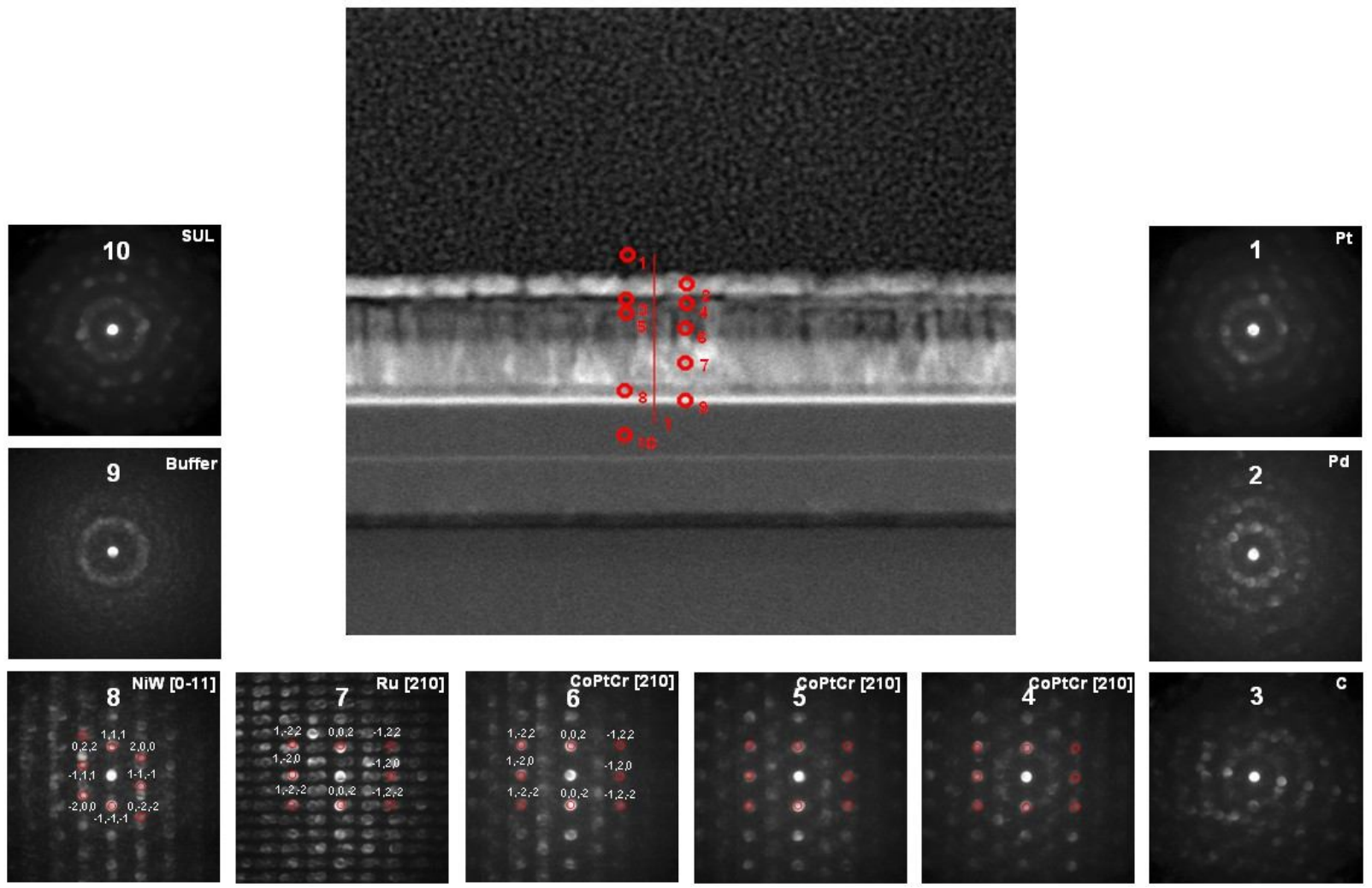

FIG. 2. Cross-section STEM micrograph and a series of energy filtered nano-diffraction patterns along the CoPtCr film stack growth direction. 